\title{
Multi-Lah numbers and multi-Stirling numbers of the first kind
}

\author{
Dae San Kim', Hye Kyung Kim², Taekyun Kim³ ${ }^{3 *}$ Hyunseok Lee ${ }^{3}$ and Seongho Park ${ }^{3}$
}

\section{"Correspondence:}

taekyun64@hotmail.com

${ }^{3}$ Department of Mathematics,

Kwangwoon University, Seoul,

139-701, Republic of Korea

Full list of author information is

available at the end of the article

\section{Springer}

\begin{abstract}
In this paper, we introduce multi-Lah numbers and multi-Stirling numbers of the first kind and recall multi-Bernoulli numbers, all of whose generating functions are given with the help of multiple logarithm. The aim of this paper is to study several relations among those three kinds of numbers. In more detail, we represent the multi-Bernoulli numbers in terms of the multi-Stirling numbers of the first kind and vice versa, and the multi-Lah numbers in terms of multi-Stirling numbers. In addition, we deduce a recurrence relation for multi-Lah numbers.
\end{abstract}

MSC: $11 \mathrm{~B} 68 ; 11 \mathrm{~B} 73 ; 11 \mathrm{~B} 83$

Keywords: Multi-Lah numbers; Multi-Stirling numbers of the first kind; Multi-Bernoulli numbers; Multiple logarithm

\section{Introduction}

As is well known, the unsigned Stirling number $\left[\begin{array}{l}n \\ r\end{array}\right]$ counts the number of permutations of a set with $n$ elements which are products of $r$ disjoint cycles. We generalize these numbers to the multi-Stirling numbers of the first kind $S_{1}^{\left(k_{1}, k_{2}, \ldots, k_{r}\right)}(n, r)$ (see (10)) which reduce to the unsigned Stirling numbers of the first kind for $\left(k_{1}, k_{2}, \ldots, k_{r}\right)=(1,1, \ldots, 1)$. Indeed, $S_{1}^{(1,1, \ldots, 1)}(n, r)=\left[\begin{array}{l}n \\ r\end{array}\right]$.

It is also well known that the unsigned Lah number $L(n, k)$ counts the number of ways a set of $n$ elements can be partitioned into $k$ nonempty linearly ordered subsets. These numbers are generalized to the multi-Lah numbers $L^{\left(k_{1}, k_{2}, \ldots, k_{r}\right)}(n, r)$ (see (18)) which reduce to the unsigned Lah numbers for $\left(k_{1}, k_{2}, \ldots, k_{r}\right)=(1,1, \ldots, 1)$. In fact, $L^{(1,1, \ldots, 1)}(n, r)=L(n, r)$.

In addition, we need to recall the multi-Bernoulli numbers $B_{n}^{\left(k_{1}, k_{2}, \ldots, k_{r}\right)}($ see (9)) which were introduced earlier under the different name of generalized Bernoulli numbers of order $r$ in [7]. These numbers reduce to the Bernoulli numbers of order $r$ up to some constants. Indeed, we see that $B_{m}^{(1,1, \ldots, 1)}=\frac{1}{r !}(-1)^{m} B_{m}^{(r)}$.

The common feature of those three kinds of numbers is that they are all defined with the help of the multiple logarithm $\mathrm{Li}_{k_{1}, k_{2}, \ldots, k_{r}}(z)$ (see (8)), which reduces to the polylogarithm $\mathrm{Li}_{k_{1}}(z)$, for $r=1$.

The aim of this paper is to study several relations among those three kinds of numbers. In more detail, we represent the multi-Bernoulli numbers in terms of the multi-Stirling numbers of the first kind and vice versa, and the multi-Lah numbers in terms of multi-

(c) The Author(s) 2021. This article is licensed under a Creative Commons Attribution 4.0 International License, which permits use sharing, adaptation, distribution and reproduction in any medium or format, as long as you give appropriate credit to the original author(s) and the source, provide a link to the Creative Commons licence, and indicate if changes were made. The images or other third party material in this article are included in the article's Creative Commons licence, unless indicated otherwise in a credit line to the material. If material is not included in the article's Creative Commons licence and your intended use is not permitted by statutory regulation or exceeds the permitted use, you will need to obtain permission directly from the copyright holder. To view a copy of this licence, visit http://creativecommons.org/licenses/by/4.0/. 
Stirling numbers. Moreover, we deduce a recurrence relation for multi-Lah numbers. For the rest of this section, we recall the necessary facts that will be needed throughout this paper.

The unsigned Stirling numbers $L(n, k)$ are defined by

$$
\langle x\rangle_{n}=\sum_{k=0}^{n} L(n, k)(x)_{k}, \quad n \geq 0(\text { see }[2-6]),
$$

where $\langle x\rangle_{0}=1,\langle x\rangle_{n}=x(x+1) \cdots(x+n-1), n \geq 1$, and $(x)_{0}=1,(x)_{n}=x(x-1) \cdots(x-n+$ $1), n \geq 1$.

The inverse formula of (1) is given by

$$
(x)_{n}=\sum_{k=0}^{n}(-1)^{n-k} L(n, k)\langle x\rangle_{k}, \quad n \geq 0 .
$$

From (1), we can derive the generating function of unsigned Lah numbers given by

$$
\frac{1}{k !}\left(\frac{t}{1-t}\right)^{k}=\sum_{n=k}^{\infty} L(n, k) \frac{t^{n}}{n !} \quad(\text { see }[2,5,8]) .
$$

Thus, we note that

$$
L(n, k)=\left(\begin{array}{c}
n-1 \\
k-1
\end{array}\right) \frac{n !}{k !}, \quad n, k \geq 1(\text { see }[2,8]) .
$$

The Stirling numbers of the first kind are defined by

$$
(x)_{n}=\sum_{k=0}^{n} S_{1}(n, k) x^{k}, \quad n \geq 0(\text { see }[6,8]),
$$

and the Stirling numbers of the second kind are defined by

$$
x^{n}=\sum_{k=0}^{n} S_{2}(n, k)(x)_{k}, \quad n \geq 0(\text { see }[2,5,8]) .
$$

From (4) and (5), we note that

$$
\frac{1}{k !}\left(e^{t}-1\right)^{k}=\sum_{n=k}^{\infty} S_{2}(n, k) \frac{t^{n}}{n !} \quad(\text { see }[2-4,8])
$$

and

$$
\frac{1}{k !}(\log (1+t))^{k}=\sum_{n=k}^{\infty} S_{1}(n, k) \frac{t^{n}}{n !} \quad(\text { see }[8]) .
$$

For any $k_{i} \geq 1(1 \leq i \leq r)$, and $|z|<1$, the multiple logarithm is defined by

$$
\operatorname{Li}_{k_{1}, k_{2}, \ldots, k_{r}}(z)=\sum_{0<m_{1}<m_{2}<\cdots<m_{r}} \frac{z^{m_{r}}}{m_{1}^{k_{1}} m_{2}^{k_{2}} \cdots m_{r}^{k_{r}}} \quad(\text { see }[1,7]) .
$$

If $r=1, \mathrm{Li}_{k_{1}}(z)=\sum_{m=1}^{\infty} \frac{z^{m}}{m^{k_{1}}}$ is the polylogarithm. 
The multi-Bernoulli numbers, which are called the generalized Bernoulli numbers of order $r$ in [7], are defined by

$$
\left.\frac{\mathrm{Li}_{k_{1}, k_{2}, \ldots, k_{r}}(z)}{z^{r}}\right|_{z=1-e^{-t}}=\sum_{m=0}^{\infty} \frac{B_{m}^{\left(k_{1}, k_{2}, \ldots, k_{r}\right)}}{m !} t^{m}
$$

From (13), we note that

$$
\overbrace{B_{m}^{(1,1, \ldots, 1)}}^{r \text {-times }}=\frac{1}{r !}(-1)^{m} B_{m}^{(r)}, \quad m \geq 0, r \geq 1 \text { (see [7]), }
$$

where $B_{n}^{(r)}$ are the Bernoulli numbers of order $r$ given by

$$
\left(\frac{t}{e^{t}-1}\right)^{r}=\sum_{n=0}^{\infty} B_{n}^{(r)} \frac{t^{n}}{n !} \quad(\text { see }[1,7,8]) .
$$

\section{Multi-Lah numbers and multi-Stirling numbers of the first kind}

Now, we define the multi-Stirling numbers of the first kind by

$$
\operatorname{Li}_{k_{1}, k_{2}, \ldots, k_{r}}(t)=\sum_{n=r}^{\infty} S_{1}^{\left(k_{1}, k_{2}, \ldots, k_{r}\right)}(n, r) \frac{t^{n}}{n !}
$$

where $k_{i} \geq 1(1 \leq i \leq r-1), k_{r} \geq 2$, and $|t|<1$.

From (8), we note that

$$
\begin{aligned}
\frac{d}{d t} \operatorname{Li}_{k_{1}, k_{2}, \ldots, k_{r}}(t) & =\frac{d}{d t} \sum_{0<m_{1}<m_{2}<\cdots<m_{r}} \frac{t^{m_{r}}}{m_{1}^{k_{1}} m_{2}^{k_{2}} \cdots m_{r}^{k_{r}}} \\
& =\frac{1}{t} \sum_{0<m_{1}<m_{2}<\cdots<m_{r}} \frac{t^{m_{r}}}{m_{1}^{k_{1}} m_{2}^{k_{2}} \cdots m_{r-1}^{k_{r-1}} m_{r}^{k_{r}-1}} \\
& =\frac{1}{t} \operatorname{Li}_{k_{1}, k_{2}, \ldots, k_{r-1}, k_{r}-1}(t) .
\end{aligned}
$$

Let us take $k_{r}=1$ in (11). Then we have

$$
\begin{aligned}
\frac{d}{d t} \operatorname{Li}_{k_{1}, k_{2}, \ldots, k_{r-1}, 1}(t) & =\frac{1}{t} \operatorname{Li}_{k_{1}, k_{2}, \ldots, k_{r-1}, 0}(t) \\
& =\frac{1}{t} \sum_{0<m_{1}<\cdots<m_{r-1}} \frac{1}{m_{1}^{k_{1}} \cdots m_{r-1}^{k_{r-1}}} \sum_{m_{r}=m_{r-1}+1}^{\infty} t^{m_{r}} \\
& =\sum_{0<m_{1}<m_{2}<\cdots<m_{r-1}} \frac{1}{m_{1}^{k_{1}} m_{2}^{k_{2}} \cdots m_{r-1}^{k_{r-1}}} \frac{t^{m_{r-1}+1}}{1-t} \frac{1}{t} \\
& =\frac{1}{1-t} \operatorname{Li}_{k_{1}, k_{2}, \ldots, k_{r-1}}(t) .
\end{aligned}
$$


We claim that the following relations hold. For this, we only need to show the first equality which we prove by induction on $r$ :

$$
\mathrm{Li}_{\underbrace{1,1, \ldots, 1}_{r \text {-times }}}(t)=\frac{1}{r !}(-\log (1-t))^{r}=\sum_{n=r}^{\infty}(-1)^{n-r} S_{1}(n, r) \frac{t^{n}}{n !} .
$$

If $r=1$, then $\operatorname{Li}_{1}(t)=\sum_{m=1}^{\infty} \frac{t^{m}}{m}=-\log (1-t)$, as we wanted. Assume that $r \geq 2$ and that the relationship holds for $r-1$. By (12) and induction hypothesis, we get

$$
\frac{d}{d t} \operatorname{Li}_{\underbrace{1,1, \ldots, 1}_{r \text {-times }}}(t)=\frac{1}{1-t} \operatorname{Li}_{\underbrace{1,1, \ldots, 1}_{(r-1) \text {-times }}}(t)=\frac{1}{(r-1) !} \frac{1}{1-t}(-\log (1-t))^{r-1} .
$$

Now, by (14) we obtain

$$
\begin{aligned}
\underbrace{\operatorname{Li}_{1,1, \ldots, 1}(t)}_{r \text {-times }} & =\frac{1}{(r-1) !} \int_{0}^{t} \frac{1}{1-t}(-\log (1-t))^{r-1} d t \\
& =\frac{1}{(r-1) !} \int_{0}^{-\log (1-t)} u^{r-1} d u=\frac{1}{r !}(-\log (1-t))^{r} .
\end{aligned}
$$

Thus our proof is completed. From (10), we note that

$$
\operatorname{Li}_{\underbrace{1,1, \ldots, 1}_{r \text {-times }}}(t)=\sum_{n=r}^{\infty} \overbrace{S_{1}^{(1,1, \ldots, 1)}}^{r \text {-times }}(n, r) \frac{t^{n}}{n !} .
$$

Therefore, by (13) and (16), we obtain the following lemma.

Lemma 1 For $n, r \geq 1$, we have

$$
\overbrace{1}^{(1,1, \ldots, 1)}(n, r)=(-1)^{n-r} S_{1}(n, r)=\left[\begin{array}{l}
n \\
r
\end{array}\right] \text {, }
$$

where $\left[\begin{array}{l}n \\ r\end{array}\right]$ are the unsigned Stirling numbers of the first kind.

We observe that

$$
\begin{aligned}
& \frac{\operatorname{Li}_{k_{1}, k_{2}, \ldots, k_{r}}\left(1-e^{-t}\right)}{\left(1-e^{-t}\right)^{r}} \\
& \quad=\frac{1}{\left(1-e^{-t}\right)^{r}} \sum_{m=r}^{\infty} S_{1}^{\left(k_{1}, k_{2}, \ldots, k_{r}\right)}(m, r) \frac{1}{m !}\left(1-e^{-t}\right)^{m} \\
& =\frac{1}{\left(1-e^{-t}\right)^{r}} \sum_{l=r}^{\infty} \sum_{m=r}^{l}(-1)^{m-l} S_{2}(l, m) S_{1}^{\left(k_{1}, k_{2}, \ldots, k_{r}\right)}(m, r) \frac{t^{l}}{l !} \\
& =\frac{(-1)^{r} t^{r}}{\left(1-e^{-t}\right)^{r}} \sum_{l=0}^{\infty} \sum_{m=r}^{l+r}(-1)^{m-l} S_{2}(l+r, m) S_{1}^{\left(k_{1}, \ldots, k_{r}\right)}(m, r) \frac{l !}{(l+r) !} \frac{t^{l}}{l !}
\end{aligned}
$$




$$
\begin{aligned}
& =\sum_{j=0}^{\infty} B_{j}^{(r)}(-1)^{r-j} \frac{t^{j}}{j !} \sum_{l=0}^{\infty}\left(\sum_{m=r}^{l+r}(-1)^{m-l} S_{2}(l+r, m) S_{1}^{\left(k_{1}, \ldots, k_{r}\right)}(m, r) \frac{1}{r !\left(\begin{array}{c}
(+r \\
l
\end{array}\right)}\right) \frac{t^{l}}{l !} \\
& =\sum_{n=0}^{\infty}\left(\sum_{l=0}^{n} \sum_{m=r}^{l+r} \frac{\left(\begin{array}{c}
n \\
l
\end{array}\right) B_{n-l}^{(r)}(-1)^{n-r-m}}{r !\left(\begin{array}{c}
l+r \\
r
\end{array}\right)} S_{2}(l+r, m) S_{1}^{\left(k_{1}, \ldots, k_{r}\right)}(m, r)\right) \frac{t^{n}}{n !} .
\end{aligned}
$$

Therefore, by (9) and (17), we obtain the following theorem.

Theorem 2 For $k_{i} \geq 1(i=1,2, \ldots, r)$, and $n \geq 0$, we have

$$
B_{n}^{\left(k_{1}, k_{2}, \ldots, k_{r}\right)}=\sum_{l=0}^{n} \sum_{m=r}^{l+r} \frac{\left(\begin{array}{l}
n \\
l
\end{array}\right) B_{n-l}^{(r)}(-1)^{n-r-m}}{r !\left(\begin{array}{c}
l+r \\
r
\end{array}\right)} S_{2}(l+r, m) S_{1}^{\left(k_{1}, \ldots, k_{r}\right)}(m, r) .
$$

For any integer $k_{i}(i=1,2, \ldots, r)$, in the view of (9), we define $L^{\left(k_{1}, k_{2}, \ldots, k_{r}\right)}(n, r)$ for $n, r \geq 0$, which are called multi-Lah numbers, as

$$
\frac{\operatorname{Li}_{k_{1}, k_{2}, \ldots, k_{r}}\left(1-e^{-t}\right)}{(1-t)^{r}}=\sum_{n=r}^{\infty} L^{\left(k_{1}, k_{2}, \ldots, k_{r}\right)}(n, r) \frac{t^{n}}{n !}
$$

From (13), we note that

$$
\begin{aligned}
\sum_{n=r}^{\infty} \overbrace{(1,1, \ldots, 1)}^{r \text {-times }}(n, r) \frac{t^{n}}{n !} & =\frac{1}{(1-t)^{r}} \operatorname{Li}_{\underbrace{1,1, \ldots, 1}_{r \text {-times }}}\left(1-e^{-t}\right) \\
& =\frac{1}{(1-t)^{r}} \frac{1}{r !}\left(-\log \left(1-\left(1-e^{-t}\right)\right)\right)^{r} \\
& =\frac{1}{r !}\left(\frac{t}{1-t}\right)^{r}=\sum_{n=r}^{\infty} L(n, r) \frac{t^{n}}{n !} .
\end{aligned}
$$

Thus, by (19), we get

$$
\overbrace{}^{\overbrace{(1,1, \ldots, 1)}^{r \text {-times }}}(n, r)=L(n, r), \quad n, r \geq 0
$$

For $n \geq 1$, from (8) and (18), we get

$$
\begin{aligned}
& \sum_{n=r}^{\infty} L^{\left(k_{1}, \ldots, k_{r-1},-k_{r}\right)}(n, r) \frac{t^{n}}{n !} \\
& \quad=\frac{1}{(1-t)^{r}} \operatorname{Li}_{k_{1}, \ldots, k_{r-1},-k_{r}}\left(1-e^{t}\right) \\
& \quad=\frac{1}{(1-t)^{r}} \sum_{0<m_{1}<m_{2}<\cdots<m_{r-1}} \frac{1}{m_{1}^{k_{1}} m_{2}^{k_{2}} \cdots m_{r-1}^{k_{r}}} \sum_{m_{r}=m_{r-1}+1}^{\infty} \frac{\left(1-e^{-t}\right)^{m_{r}}}{m_{r}^{-k_{r}}} \\
& \quad=\frac{1}{(1-t)^{r}} \sum_{0<m_{1}<m_{2}<\cdots<m_{r-1}} \frac{1}{m_{1}^{k_{1}} m_{2}^{k_{2}} \cdots m_{r-1}^{k_{r-1}}} \sum_{m_{r}=1}^{\infty} \frac{\left(1-e^{-t}\right)^{m_{r}+m_{r-1}}}{\left(m_{r}+m_{r-1}\right)^{-k_{r}}} \\
& =\frac{1}{(1-t)^{r}} \sum_{0<m_{1}<m_{2}<\cdots<m_{r-1}} \frac{\left(1-e^{t}\right)^{m_{r-1}}}{m_{1}^{k_{1}} m_{2}^{k_{2}} \cdots m_{r-1}^{k_{r-1}}} \sum_{m_{r}=1}^{\infty} \frac{(-1)^{m_{r}} m_{r} !}{\left(m_{r}+m_{r-1}\right)^{-k_{r}}} \frac{1}{m_{r} !}\left(e^{-t}-1\right)^{m_{r}}
\end{aligned}
$$




$$
\begin{aligned}
= & \frac{1}{(1-t)^{r}} \sum_{0<m_{1}<m_{2}<\cdots<m_{r-1}} \frac{\left(1-e^{t}\right)^{m_{r-1}}}{m_{1}^{k_{1}} m_{2}^{k_{2}} \cdots m_{r-1}^{k_{r-1}}} \sum_{m_{r}=1}^{\infty} \frac{(-1)^{m_{r}} m_{r} !}{\left(m_{r}+m_{r-1}\right)^{-k_{r}}} \sum_{l=m_{r}}^{\infty} S_{2}\left(l, m_{r}\right) \frac{(-t)^{l}}{l !} \\
= & \frac{1}{(1-t)^{r}} \sum_{0<m_{1}<m_{2}<\cdots<m_{r-1}} \frac{\left(1-e^{t}\right)^{m_{r-1}}}{m_{1}^{k_{1}} m_{2}^{k_{2}} \cdots m_{r-1}^{k_{r-1}}} \sum_{l=1}^{\infty}\left(\sum_{m_{r}=1}^{l} \frac{(-1)^{m_{r}+l} m_{r} !}{\left(m_{r}+m_{r-1}\right)^{-k_{r}}} S_{2}\left(l, m_{r}\right)\right) \frac{t^{l}}{l !} \\
= & \frac{1}{1-t} \sum_{l=1}^{\infty}\left(\sum_{m_{r}=1}^{l}(-1)^{m_{r}+l} m_{r} ! S_{2}\left(l, m_{r}\right) \sum_{j=0}^{k_{r}}\left(\begin{array}{c}
k_{r} \\
j
\end{array}\right) m_{r}^{k_{r}-j}\right. \\
& \left.\times\left(\frac{1}{1-t}\right)^{r-1} \sum_{0<m_{1}<m_{2}<\cdots<m_{r-1}} \frac{\left(1-e^{-t}\right)^{m_{r-1}}}{m_{1}^{k_{1}} \cdots m_{r-1}^{k_{r-1}-j}}\right) \frac{t^{l}}{l !} \\
& \frac{1}{1-t} \sum_{l=1}^{\infty}\left(\sum_{m_{r}=1}^{l}(-1)^{m_{r}+l} m_{r} ! S_{2}\left(l, m_{r}\right)\right. \\
& \times \sum_{j=0}^{k_{r}}\left(\begin{array}{l}
k_{r} \\
j
\end{array}\right)_{\left.m_{r}^{k_{r}-j}\left(\frac{1}{1-t}\right)^{r-1} \mathrm{Li}_{k_{1}, \ldots, k_{r-1}-j}\left(1-e^{-t}\right)\right) \frac{t^{l}}{l !} .}
\end{aligned}
$$

From (20), we note that

$$
\begin{aligned}
& \sum_{n=r}^{\infty} L^{\left(k_{1}, k_{2}, \ldots, k_{r-1},-k_{r}\right)}(n, r) \frac{t^{n}}{n !} \\
&=\frac{1}{1-t} \sum_{l=1}^{\infty}\left(\sum_{m_{r}=1}^{l} \sum_{j=0}^{k_{r}}(-1)^{m_{r}+l} m_{r} ! S_{2}\left(l, m_{r}\right)\left(\begin{array}{c}
k_{r} \\
j
\end{array}\right) m_{r}^{k_{r}-j}\right. \\
&\left.\times \sum_{m=r-1}^{\infty} L^{\left(k_{1}, k_{2}, \ldots, k_{r-2}, k_{r-1}-j\right)}(m, r-1) \frac{t^{m}}{m !}\right) \frac{t^{l}}{l !} \\
&= \frac{1}{1-t} \sum_{p=r}^{\infty}\left(\sum_{l=1}^{p+1-r}\left(\begin{array}{c}
p \\
l
\end{array}\right) \sum_{m_{r}=1}^{p} \sum_{j=0}^{k_{r}}(-1)^{m_{r}+l} m_{r} ! m_{r}^{k_{r}-j} S_{2}\left(l, m_{r}\right)\left(\begin{array}{c}
k_{r} \\
j
\end{array}\right)\right. \\
&\left.\times L^{\left(k_{1}, k_{2}, \ldots, k_{r-2}, k_{r-1}-j\right)}(p-l, r-1)\right) \frac{t^{p}}{p !} \\
&= \sum_{n=r}^{\infty}\left(\sum_{p=r}^{n} \sum_{l=1}^{p+1-r}\left(\begin{array}{c}
p \\
l
\end{array}\right) \sum_{m_{r}=1}^{p} \sum_{j=0}^{k_{r}}(-1)^{m_{r}+l} m_{r} ! m_{r}^{k_{r}-j} S_{2}\left(l, m_{r}\right)\left(\begin{array}{c}
k_{r} \\
j
\end{array}\right)\right. \\
&\left.\times L^{\left(k_{1}, k_{2}, \ldots, k_{r-2}, k_{r-1}-j\right)}(p-l, r-1) \frac{n !}{p !}\right) \frac{t^{n}}{n !} .
\end{aligned}
$$

Therefore, by comparing the coefficients on both sides of (21), we obtain the following theorem.

Theorem 3 For any $k_{i} \geq 1(i=1,2, \ldots, r)$, and $n, r \in \mathbb{N}$, we have

$$
\begin{aligned}
& L^{\left(k_{1}, k_{2}, \ldots, k_{r-1},-k_{r}\right)}(n, r) \\
& \quad=\sum_{p=r}^{n} \sum_{l=1}^{p} \sum_{m_{r}=1}^{p} \sum_{j=0}^{k_{r}}(-1)^{m_{r}+l} m_{r} ! m_{r}^{k_{r}-j}\left(\begin{array}{c}
p \\
l
\end{array}\right)\left(\begin{array}{c}
k_{r} \\
j
\end{array}\right)
\end{aligned}
$$




$$
\times S_{2}\left(l, m_{r}\right) L^{\left(k_{1}, k_{2}, \ldots, k_{r-2}, k_{r-1}-j\right)}(p-l, r-1) \frac{n !}{p !}
$$

Replacing $t$ by $-\log (1-t)$ in (9), we get

$$
\begin{aligned}
\frac{1}{t^{r}} \operatorname{Li}_{k_{1}, k_{2}, \ldots, k_{r}}(t) & =\sum_{m=0}^{\infty} B_{m}^{\left(k_{1}, k_{2}, \ldots, k_{r}\right)}(-1)^{m} \frac{1}{m !}(\log (1-t))^{m} \\
& =\sum_{m=0}^{\infty} B_{m}^{\left(k_{1}, k_{2}, \ldots, k_{r}\right)}(-1)^{m} \sum_{n=m}^{\infty} S_{1}(n, m)(-1)^{n} \frac{t^{n}}{n !} \\
& =\sum_{n=0}^{\infty}\left(\sum_{m=0}^{n}(-1)^{n-m} B_{m}^{\left(k_{1}, k_{2}, \ldots, k_{r}\right)} S_{1}(n, m)\right) \frac{t^{n}}{n !}
\end{aligned}
$$

On the other hand, by (10), we get

$$
\begin{aligned}
\frac{1}{t^{r}} \operatorname{Li}_{k_{1}, k_{2}, \ldots, k_{r}}(t) & =\frac{1}{t^{r}} \sum_{n=r}^{\infty} S_{1}^{\left(k_{1}, k_{2}, \ldots, k_{r}\right)}(n, r) \frac{t^{n}}{n !} \\
& =\sum_{n=0}^{\infty} S_{1}^{\left(k_{1}, k_{2}, \ldots, k_{r}\right)}(n+r, r) \frac{n !}{(n+r) !} \frac{t^{n}}{n !} \\
& =\sum_{n=0}^{\infty} \frac{S_{1}^{\left(k_{1}, k_{2}, \ldots, k_{r}\right)}(n+r, r)}{r !\left(\begin{array}{c}
n+r \\
n
\end{array}\right)} \frac{t^{n}}{n !} .
\end{aligned}
$$

Therefore, by (22) and (23), we obtain the following theorem.

Theorem 4 For each $k_{i} \geq 1(i=1,2, \ldots, r), n \geq 0$, and $r \in \mathbb{N}$, we have

$$
S_{1}^{\left(k_{1}, k_{2}, \ldots, k_{r}\right)}(n+r, r)=r !\left(\begin{array}{c}
n+r \\
n
\end{array}\right) \sum_{m=0}^{n} B_{m}^{\left(k_{1}, k_{2}, \ldots, k_{r}\right)}\left[\begin{array}{c}
n \\
m
\end{array}\right] .
$$

Now, we observe that

$$
\begin{aligned}
& \sum_{n=r}^{\infty} L^{\left(k_{1}, k_{2}, \ldots, k_{r}\right)}(n, r) \frac{t^{n}}{n !} \\
& \quad=\frac{1}{(1-t)^{r}} \operatorname{Li}_{k_{1}, k_{2}, \ldots, k_{r}}\left(1-e^{-t}\right) \\
& \quad=\sum_{j=0}^{\infty}\left(\begin{array}{c}
r+j-1 \\
j
\end{array}\right) t^{j} \sum_{m=r}^{\infty} S_{1}^{k_{1}, k_{2}, \ldots, k_{r}}(m, r) \frac{1}{m !}\left(1-e^{-t}\right)^{m} \\
& \quad=\sum_{j=0}^{\infty}\left(\begin{array}{c}
r+j-1 \\
j
\end{array}\right) t^{j} \sum_{l=r}^{\infty}\left(\sum_{m=r}^{l} S_{1}^{\left(k_{1}, \ldots, k_{r}\right)}(m, r)(-1)^{m-l} S_{2}(l, m)\right) \frac{t^{l}}{l !} \\
& \quad=\sum_{n=r}^{\infty}\left(\sum_{l=r}^{n} \sum_{m=r}^{l} S_{1}^{\left(k_{1}, \ldots, k_{r}\right)}(m, r)(-1)^{m-l} S_{2}(l, m)\left(\begin{array}{c}
r+n-l-1 \\
n-l
\end{array}\right) \frac{n !}{l !}\right) \frac{t^{n}}{n !}
\end{aligned}
$$

Therefore, by comparing the coefficients on both sides of (24), we obtain the following theorem. 
Theorem 5 For each $k_{i}(i=1,2, \ldots, r)$, and $n, r \in \mathbb{N}$, with $n \geq r$, we have

$$
\begin{aligned}
& L^{\left(k_{1}, k_{2}, \ldots, k_{r}\right)}(n, r) \\
& \quad=\sum_{l=r}^{n} \sum_{m=r}^{l} S_{1}^{\left(k_{1}, \ldots, k_{r}\right)}(m, r)(-1)^{m-l} S_{2}(l, m)\left(\begin{array}{c}
r+n-l-1 \\
n-l
\end{array}\right) \frac{n !}{l !} .
\end{aligned}
$$

\section{Conclusion}

There are various ways of studying special polynomials and numbers which include generating functions, combinatorial methods, $p$-adic analysis, umbral calculus, special functions, differential equations, and probability theory. In this paper, using the generating function method and by making use of the multiple logarithm, we studied three kinds of numbers, namely the multi-Stirling numbers of the first kind, the multi-Lah numbers, and the multi-Bernoulli numbers, which reduce respectively to the unsigned Stirling numbers of the first kind, the Lah numbers, and the higher-order Bernoulli numbers up to constants when the index is specialized to $\left(k_{1}, k_{2}, \ldots, k_{r}\right)=(1,1, \ldots, 1)$. We deduced several relations among those numbers. In more detail, we expressed the multi-Bernoulli numbers in terms of the multi-Stirling numbers of the first kind and vice versa, and the multi-Lah numbers in terms of multi-Stirling numbers. Further, we derived a recurrence relation for multi-Lah numbers.

It is our continuous interest to explore some special numbers and polynomials by using different tools like those mentioned above.

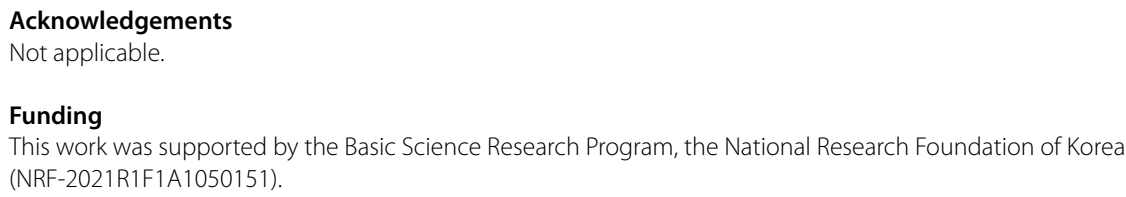

Availability of data and materials

Not applicable.

Competing interests

The authors declare that they have no competing interests.

Authors' contributions

DSK, HKK, and TK conceived of the framework and structured the whole paper; TK and DSK wrote the paper; DSK, HKK, and TK completed the revision of the article; HL and SP checked the errors of the article. All authors have read and agreed to the published version of the manuscript.

\section{Author details}

${ }^{1}$ Department of Mathematics, Sogang University, Seoul, 121-742, Republic of Korea. ${ }^{2}$ Department of Mathematical Education, Daegu Catholic University, Gyeongsan, 38430, Republic of Korea. ${ }^{3}$ Department of Mathematics, Kwangwoon University, Seoul, 139-701, Republic of Korea.

\section{Publisher's Note}

Springer Nature remains neutral with regard to jurisdictional claims in published maps and institutional affiliations.

Received: 21 June 2021 Accepted: 26 August 2021 Published online: 08 September 2021

\section{References}

1. Borwein, J.M., Chan, O.-Y.: Duality in tails of multiple-zeta values. Int. J. Number Theory 6(3), 501-514 (2010)

2. Comtet, L.: Advanced Combinatorics, the Art of Finite and Infinite Expansions, Revised and enlarged edn. Reidel, Dordrecht (1974)

3. Kim, D.S., Kim, T.: Lah-Bell numbers and polynomials. Proc. Jangjeon Math. Soc. 23(4), 577-586 (2020)

4. Kim, D.S., Kim, T.: A note on a new type of degenerate Bernoulli numbers. Russ. J. Math. Phys. 27(2), 227-235 (2020) 
5. Kim, D.S., Kim, T:: r-extended Lah-Bell numbers and polynomials associated with $r$-Lah numbers. Proc. Jangjeon Math. Soc. 24(1), 1-10 (2021)

6. Kim, H.K.: Degenerate Lah-Bell polynomials arising from degenerate Sheffer sequences. Adv. Differ. Equ. 2020, Article ID 687 (2020)

7. Kim, M.-S., Kim, T.: An explicit formula on the generalized Bernoulli number with order $n$. Indian J. Pure Appl. Math. 31(11), 1455-1461 (2000)

8. Roman, S.: The Umbral Calculus. Pure and Applied Mathematics, vol. 111. Academic Press, New York (1984)

Submit your manuscript to a SpringerOpen ${ }^{\odot}$ journal and benefit from:

- Convenient online submission

Rigorous peer review

- Open access: articles freely available online

- High visibility within the field

- Retaining the copyright to your article

Submit your next manuscript at $\gg$ springeropen.com 